\title{
Inflammatory Cytokines in Maternal Circulation and Placenta of Chromosomally Abnormal First Trimester Miscarriages
}

\author{
Jean Calleja-Agius, ${ }^{1,2}$ Eric Jauniaux, ${ }^{1}$ and Shanthi Muttukrishna ${ }^{3}$ \\ ${ }^{1}$ UCL EGA Institute for Women's Health, University College London, 86-96, Chenies Mews, London, WCIE 6HX, UK \\ ${ }^{2}$ Department of Anatomy, Faculty of Medicine and Surgery, University of Malta, Tal-Qroqq, Msida 2090, Malta \\ ${ }^{3}$ Anu Research Centre, UCC Department of Obstetrics and Gynaecology, Cork University Maternity Hospital, \\ 5th Floor, Wilton, Cork, Ireland \\ Correspondence should be addressed to Jean Calleja-Agius, jean.calleja-agius@um.edu.mt
}

Received 7 June 2011; Accepted 3 August 2011

Academic Editor: Gilbert Faure

Copyright ( $\odot 2012$ Jean Calleja-Agius et al. This is an open access article distributed under the Creative Commons Attribution License, which permits unrestricted use, distribution, and reproduction in any medium, provided the original work is properly cited.

\begin{abstract}
The impact of abnormal placental karyotype on the inflammatory response within the villous tissue and peripheral circulation of women with miscarriage was evaluated. Villous $(n=38)$ and venous blood samples $(n=26)$ were obtained from women with missed miscarriage. Tissue chromosome analysis indicated 23 abnormal and 15 normal karyotypes. Concentration of tumour necrosis factor alpha (TNF $\alpha$ ), TNF-R1 and TNF-R2, and interleukin (IL)-10 were measured using flowcytometric bead array in fresh villous homogenate, cultured villous extracts, culture medium, maternal whole blood, and plasma. Plasma TNF $\alpha /$ IL10 ratios were significantly $(P<0.05)$ lower in miscarriages with abnormal karyotype. In the abnormal karyotype group, there were significantly higher levels of TNF $\alpha(P<0.01)$, IL-10 $(P<0.01)$, TNF-R1 $(P<0.001)$, and TNF-R2 $(P<0.001)$ in the villous extracts and culture-conditioned medium compared to normal karyotype group. In miscarriage with abnormal karyotype, there is an exacerbated placental inflammatory response, in contrast to miscarriage of normal karyotype where maternal systemic response is increased.
\end{abstract}

\section{Introduction}

Early pregnancy loss is the most common pregnancy complication [1]. Around 60\% of first trimester miscarriages are associated with a chromosomal abnormality [2-4]. Most chromosomal abnormalities are associated with primary abnormal trophoblast invasion of the uterine decidua [57]. As the placental development becomes increasingly dependent on fetal synthesis towards the end of the first trimester, isolated major fetal structural defects leading to early fetal demise can also lead to secondary placental dysfunction causing a miscarriage [8].

Inflammatory processes taking place at the feto-maternal interface are essential for normal implantation in human pregnancy [9-11]. It has been suggested that the main regulator of this inflammatory reaction could be uterine natural killer (NK) cells [10]. Proinflammatory cytokines like tumour necrosis factor alpha $(\mathrm{TNF} \alpha)$ have been shown to inhibit trophoblast migration through the elevation of plasminogen activator inhibitor-1 in first trimester villous explant cultures, causing abnormal trophoblast invasion [12]. TNF $\alpha$ has also been shown to downregulate the endocrine function of trophoblasts, leading to pregnancy failure [13]. It has been suggested that a network might exist in which hormones together with cytokines regulate the decidual expression of HLA-G, an antigen preferentially expressed by trophoblast, thus, maintaining maternal-fetal tolerance [14].

There is evidence of a shift in T-helper 1 (TNF $\alpha)$ : Thelper 2 (interleukin, IL-10) ratio in the maternal circulation and placental villous tissue in first trimester miscarriages [15-17]. However, most previous studies have used placental tissue for early pregnancy failure without knowledge of the karyotype or have focused on women presenting with recurrent miscarriage, and thus little is known about the impact of the conceptus karyotype on the placental and systemic inflammatory responses in early pregnancy failure. The aim of this study was therefore to investigate the 
maternal circulatory levels, villous expression, and secretion in vitro of TNF $\alpha, \mathrm{TNF} \alpha$ receptors, and IL-10 and to evaluate the Th1 and Th2 cytokine ratio in early pregnancy failures with and without a chromosomal abnormality.

\section{Materials and Methods}

Chorionic villous samples $(n=38)$ were obtained from women presenting with a missed miscarriage, undergoing the evacuation of retained products of conception (ERPC) at University College London Hospital (UCLH). All women were nonsmokers, with normal body mass index (BMI) ranging between 20 and 30 , not on medication, and with a history of regular menstrual cycles. The date of the last menstrual period (LMP) was used to calculate the gestational age, and women with a history of recurrent miscarriage or who did not know their LMP were excluded from the study. The gestational ages at the time of ERPC ranged between 9 weeks and 0 days and 13 weeks and 6 days gestation. Ultrasound measurement of the fetal crown-rump length (CRL) was used to evaluate the time interval between fetal demise and the surgical procedure of ERPC.

In 12 cases with normal karyotype and 14 with abnormal karyotype, maternal peripheral venous blood $(10 \mathrm{~mL})$ was collected by sterile venepuncture into bottles with lithium heparin at the time of ERPC. One $\mathrm{mL}$ of uncoagulated blood was aspirated aseptically for whole blood analysis while the rest was centrifuged within 2 hours of collection, and the plasma supernatant was stored at $-80^{\circ} \mathrm{C}$ until assayed.

This study was approved by the joint UCL/UCLH ethics committee on the ethics of human research. Written informed consent was obtained from each participant at the Early Pregnancy Unit prior to the surgical uterine evacuation of retained products of conception (ERPC).

2.1. Flowcytometric Analysis of Fluorescent Antibody-Labelled Whole Blood. Initial whole blood validation experiments showed that an incubation period of 12 hours with $40 \mathrm{ng} / \mathrm{mL}$ of lipopolysaccharide (40 LPS) gave the highest increment above basal level (0 LPS) in terms of cytokine expression by the activated viable monocytes. Dual antibody labelling was carried out with specific mouse antihuman antibodies (AbD Serotec, Oxford, UK) that were conjugated to spectrally distinct fluorochromes to identify the monocyte population (CD14) positive for the cytokine/receptor of interest. The method we used has been described elsewhere [18].

2.2. In Vitro Cultures. Following the ERPC, the placental villi were separated from the rest of the POC and washed twice in sterile Hank's Balanced Salt Solution with 0.1\% Gentamycin Solution (Sigma-Aldrich, St Louis, USA) and 1\% Amphotericin B (Invitrogen, Paisley, UK) to remove any blood. A biopsy of villous tissue measuring $\sim 1 \mathrm{~cm}^{3}$ was snap frozen in liquid nitrogen and stored at $-80^{\circ} \mathrm{C}$ until homogenised to measure the cytokine/receptor content in the villous tissue on the day of evacuation (Day 0). In all cases, a villous sample was sent to a commercial cytogenetic laboratory (TDL, London, UK) within 2 hours of the ERPC, and karyotyping was carried out by standard cultur- ing, suspension harvest and G-band analysis methodology [19].

Villous tissue obtained from the ERPC was divided into equal sections under sterile conditions under a laminar hood and weighed. Each of the villous biopsies of known weight was cultured in 24-well culture plates containing $1 \mathrm{~mL}$ culture medium per well, in triplicate wells. Villous explants derived from each individual woman were cultured separately for 3 days at $6 \%$ and at $20 \%$ oxygen $\left(\mathrm{O}_{2}\right)$ levels in a moist incubator at $37^{\circ} \mathrm{C}, 5 \% \mathrm{CO}_{2}$. The culture medium was made up of D-MEM: F-12 (1:1) + GlutaMAXI (Invitrogen, Paisley, UK) with $0.1 \%$ Gentamycin Solution (Sigma-Aldrich, St Louis, USA) and 1\% Amphotericin and $1 \%$ Insulin-Transferrin-Selenium A serum supplement (Invitrogen, Paisley, UK).

Villous explant culture conditioned medium and placental villi were collected after incubation for 1 day, 2 days, and 3 days from the individual wells. Cultured villous explants were homogenized for cytokine analysis. Homogenization of villous tissue was carried out as previously described by our group [20]. All cytokines/receptors concentrations in villous explants homogenates and culture-conditioned medium were normalized against weight of tissue/well as $\mathrm{pg} / \mathrm{gram}$ villous tissue.

2.3. Cytokine and Receptor Assays. Cytokines and receptors in plasma, culture medium, and homogenised villi were measured using BD Cytometric Bead Array (CBA) Human Soluble Protein Flex Sets and BD FACSArray bioanalyzer flowcytometer following manufacturer's instructions (BD Biosciences, San Jose, California, USA). The limit of detection was $0.7 \mathrm{pg} / \mathrm{mL}$ for TNF $\alpha, 0.13 \mathrm{pg} / \mathrm{mL}$ for IL-10, $5.2 \mathrm{pg} / \mathrm{mL}$ for TNF-R1, and $1.4 \mathrm{pg} / \mathrm{mL}$ for TNF-R2. The intra-assay coefficient of variation was $10.2 \%$ for TNF $\alpha, 6.4 \%$ for IL-10, $2.6 \%$ for TNF-R1, and 7.1\% for TNF-R2. The interassay coefficient of variation was $5 \%$ for $\mathrm{TNF} \alpha, 11 \%$ for IL-10, 10.1\% for TNF-R1, and 5.6\% for TNF-R2. The results were presented in graphical and tabular formats using the FCAP Array Software (BD Biosciences, San Jose, Calif, USA).

2.4. Statistical Analysis. For villous cytokine expression and the time- and $\mathrm{O}_{2}$-dependent villous explant medium concentrations, the data were log transformed to achieve normality, which was confirmed using the Shapiro-Wilks test and Q-Q plots. Outliers were identified using Cook's distance, and those exceeding the threshold $4 / n$ were removed.

For villous in vivo cytokine expression, ANOVA was performed on the log-transformed data, to estimate means for each cytokine by karyotype, adjusted for gestation at sampling. Multivariate linear regressions were carried out on the log-transformed data, to estimate the means for each cytokine by karyotype, $\mathrm{O}_{2}$ level (6 versus $20 \%$ ), and day of culture (Day 1, 2, or 3), adjusted for gestation. For cytokine/receptor levels in the plasma and monocytes, data were normalized by log transformation, and unpaired $t$-test was carried out.

A $P$-value less than 0.05 was considered statistically significant. The statistical analysis and graphs were produced 
TABLE 1: In vivo cytokine and receptors, measured in snap frozen villous tissue collected on the day of ERPC-comparison done using 2-way ANOVA, using the variables of karyotype (normal versus abnormal) and gestation (weeks). There was no significant difference between gestation and karyotype. Estimated marginal means for each marker by karyotype, adjusted for gestation, are presented here.

\begin{tabular}{lccc}
\hline Cytokine/receptor & $\begin{array}{c}\text { Normal karyotype } \\
\text { pg/g }(95 \% \mathrm{CI})\end{array}$ & $\begin{array}{c}\text { Abnormal karyotype } \\
\text { pg/g }(95 \% \text { CI })\end{array}$ & $\begin{array}{c}\text { Normal versus abnormal } \\
\text { karyotype } \\
P \text { value }\end{array}$ \\
\hline TNF $\alpha$ & $8.2(5.3,12.6)$ & $7.2(4.2,12.2)$ & 0.92 \\
IL-10 & $2.2(1.3,3.8)$ & $2.9(1.6,5.2)$ & 0.79 \\
TNF $\alpha$ IL-10 & $4.5(2.2,9.0)$ & $2.3(1.3,4.2)$ & 0.62 \\
TNF-R1 & $2499.1(1488.8,4195.3)$ & $2753.26(1927.21,3933.4)$ & 0.56 \\
TNF-R2 & $12440.9(6876.6,22507.7)$ & $12157.9(8716.9,16957.2)$ & 0.68 \\
\hline
\end{tabular}

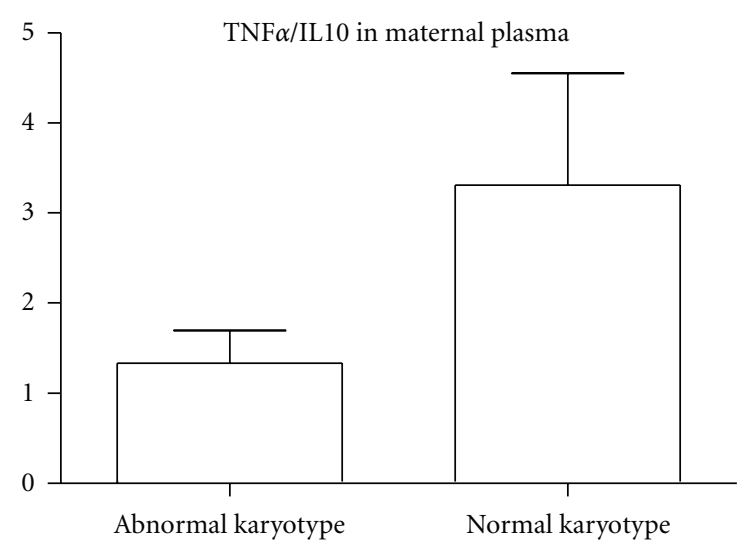

FIgURE 1: TNF $\alpha /$ IL-10 in the maternal circulation (abnormal karyotype, $n=14$; normal karyotype, $n=12$ ). There was a significantly $(P<0.05)$ lower TNF $\alpha / \mathrm{IL}-10$ ratio in the plasma of miscarriages of abnormal karyotype than in those with normal karyotype.

using SPSS v.17 (SPSS Inc., Chicago, Ill, USA) and GraphPad Prism v.5 (GraphPad Software inc., San Diego, Calif, USA).

\section{Results}

Karyotyping of the products of conception (POC) indicated 15 with normal karyotype and 23 abnormal karyotypes including 16 trisomies, 4 monosomy $\mathrm{X}$, and 3 triploidies. There was no difference in maternal BMI, age, parity, and ethnic distribution between the group with and without a chromosomal abnormality. The gestation at the time of ERPC, interval in days between estimated fetal demise and ERPC and fetal sex ratio was similar in both groups. In the cases where maternal blood was collected, karyotyping showed 12 normal karyotypes and 14 abnormal karyotypes.

3.1. Maternal Plasma Concentrations. The levels of TNF $\alpha$, TNF-R1, TNF-R2, and IL-10 were not significantly different between miscarriages with normal and abnormal karyotype. TNF $\alpha /$ IL-10 ratio in the plasma was significantly $(P<0.05)$ lower in miscarriages with an abnormal karyotype than those with normal karyotype (Figure 1).
3.2. Maternal Circulatory Monocyte Concentration. There was no significant difference between intracellular levels of TNF $\alpha$, TNF-R1, TNF-R2, and IL-10 in the monocytes in plasma samples of both groups. There was a 3 -fold higher $\%$ LPS stimulation of TNF $\alpha$ in the normal karyotype compared to the abnormal karyotype group (Figures 2 and 3 ).

3.3. Expression of Cytokine and Receptors in Villous Tissue. No significant differences were found in the cytokine and receptor levels in the snap frozen villous tissue homogenate samples between the groups presenting with normal and abnormal karyotype. These levels were similar in the different gestational subgroups, and across gestation (Table 1).

\subsection{Villous Tissue In Vitro Secretion and Content of Cytokines} and Receptors. The ANOVA test indicated that gestation has no significant impact on the relationship between cytokine/receptor levels and karyotype in culture conditions at both $\mathrm{O}_{2}$ concentrations.

Time in culture $(1,2$, or 3 days $)$ and $\mathrm{O}_{2}$ concentration (6 versus 20\%) did not affect the mean cytokine/receptor levels or TNF $\alpha / \mathrm{IL}-10$ ratio in villous homogenates or cultureconditioned medium adjusted for gestation (Table 2).

3.4.1. Villous Tissue Homogenates. Significantly higher levels of TNF $\alpha(P<0.01)$, IL-10 $(P<0.001)$, and TNF-R2 $(P<$ 0.001 were found in cultured villous extracts in the group with an abnormal karyotype compared to normal karyotype group. There was no significant difference in TNF $\alpha / \mathrm{IL}-10$ (Table 2).

3.4.2. Culture Medium of Villous Tissue Samples. Significantly higher levels of TNF $\alpha(P=0.001)$, IL-10 $(P<0.01)$, and TNF-R1 $(P<0.001)$ were found in the culture-conditioned medium of abnormal karyotype group compared to the normal karyotype group. There was no significant difference in TNF $\alpha /$ IL-10 (Table 2) between the groups.

\section{Discussion}

The results of our study indicate that, in sporadic miscarriages with a normal karyotype, there is an increased 


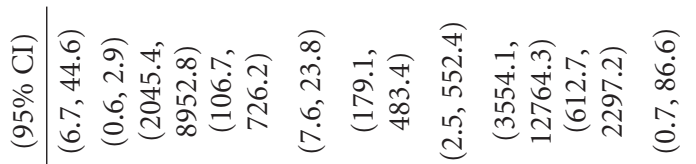

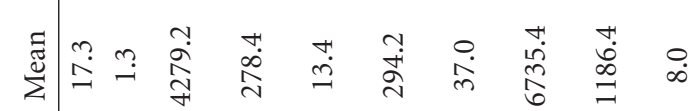

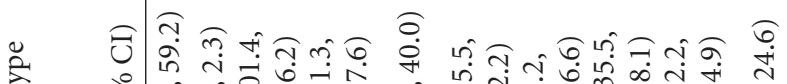

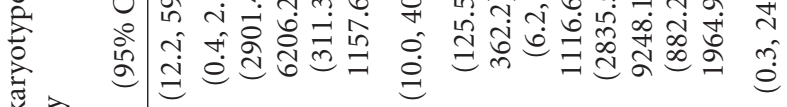
节

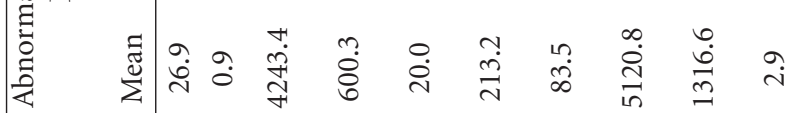

כ)

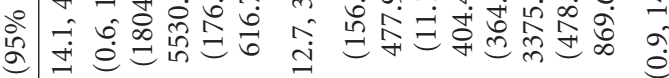

5
0
0
0
0
0
0
0
0
0
0
0
0
0

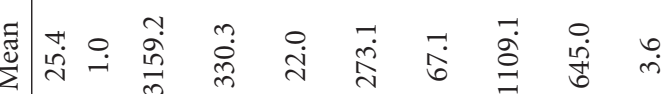

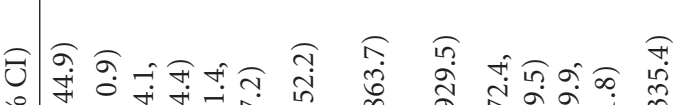

म

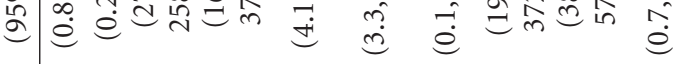

离

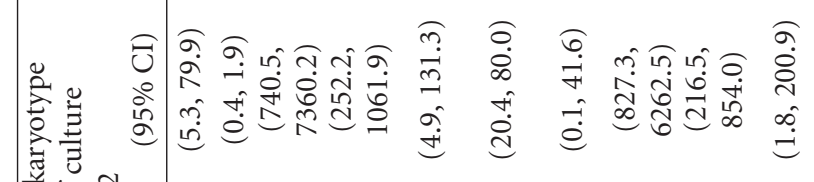

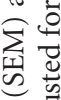

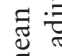

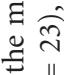

प्र००

苛

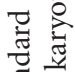

羟

芩

है तี

은

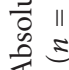

¿

武苍

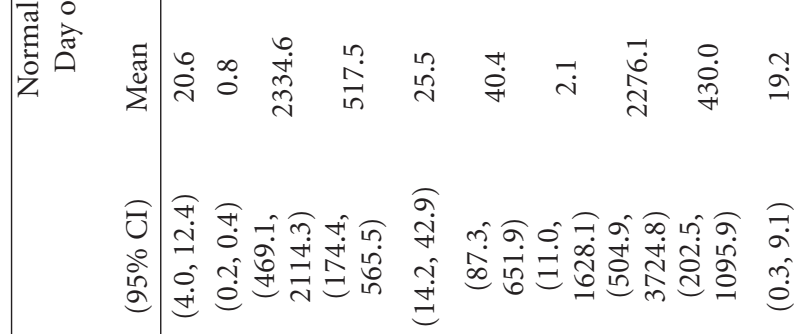

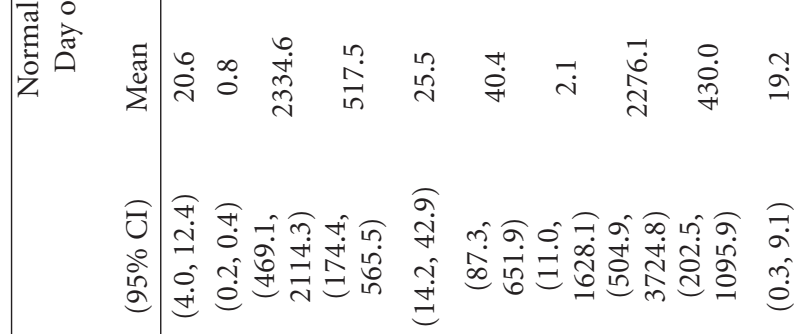

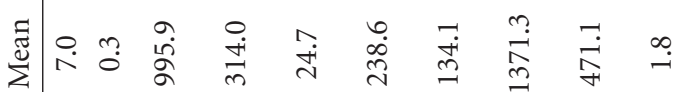
西

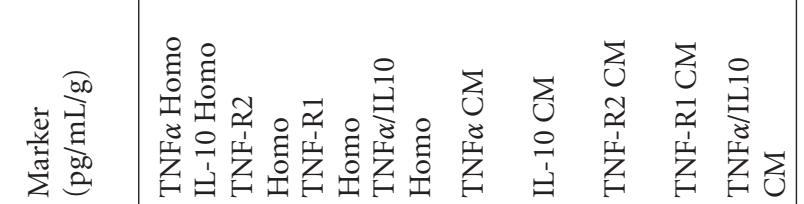

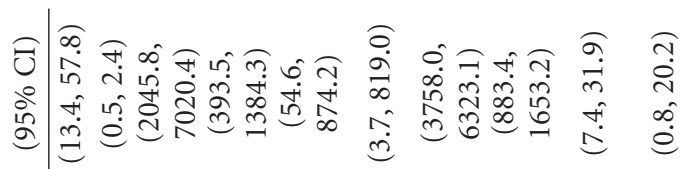

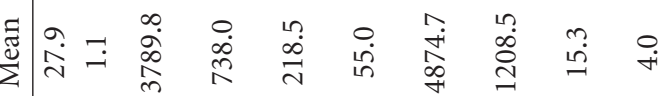

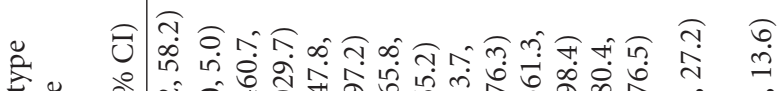

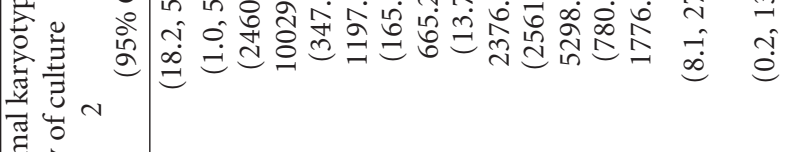

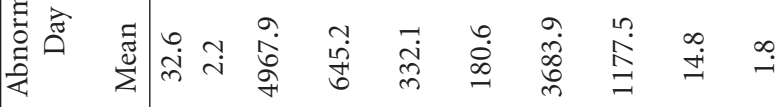

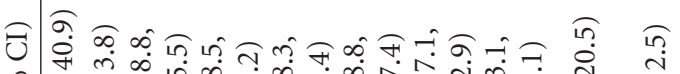

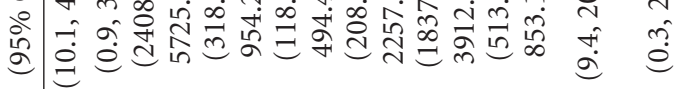
$-$

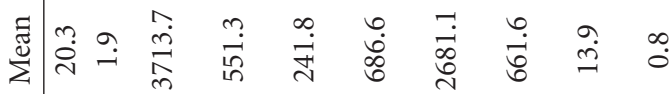

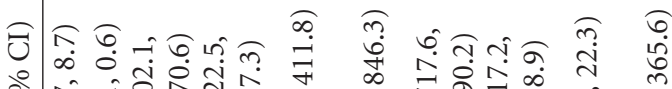

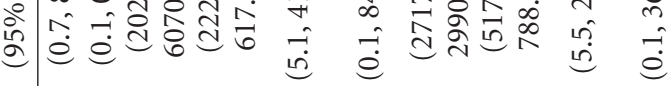

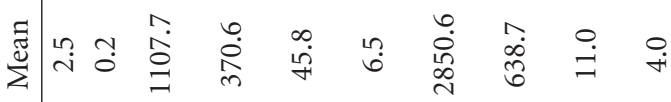

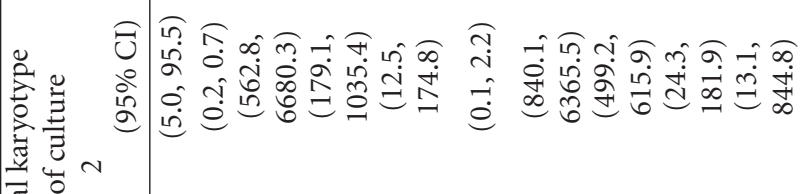

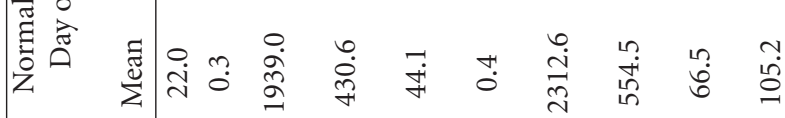

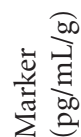

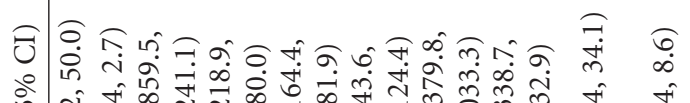

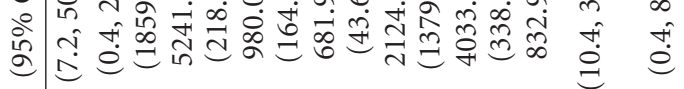

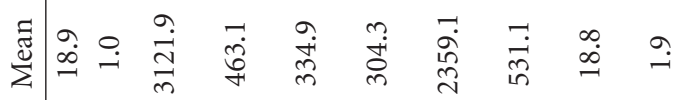

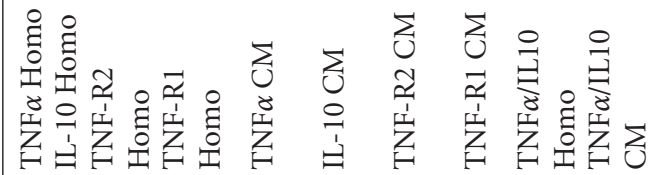




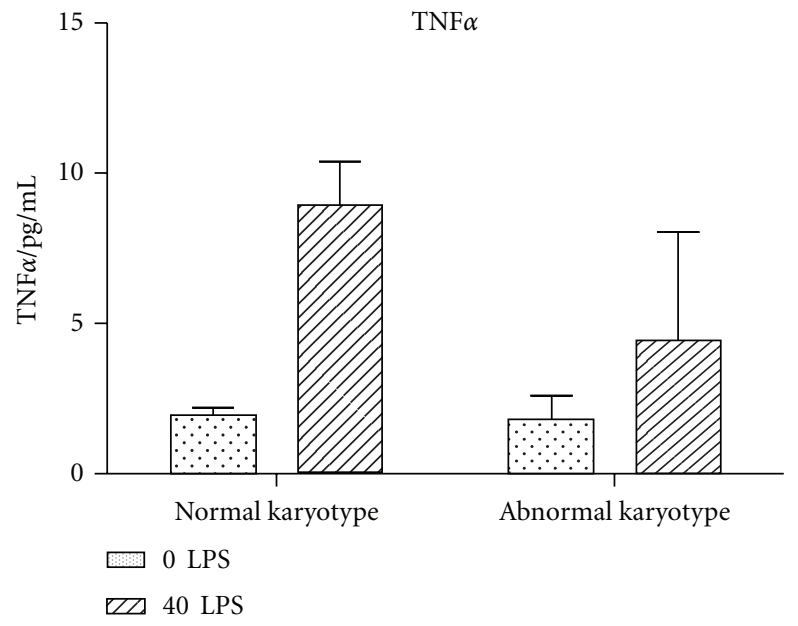

(a)

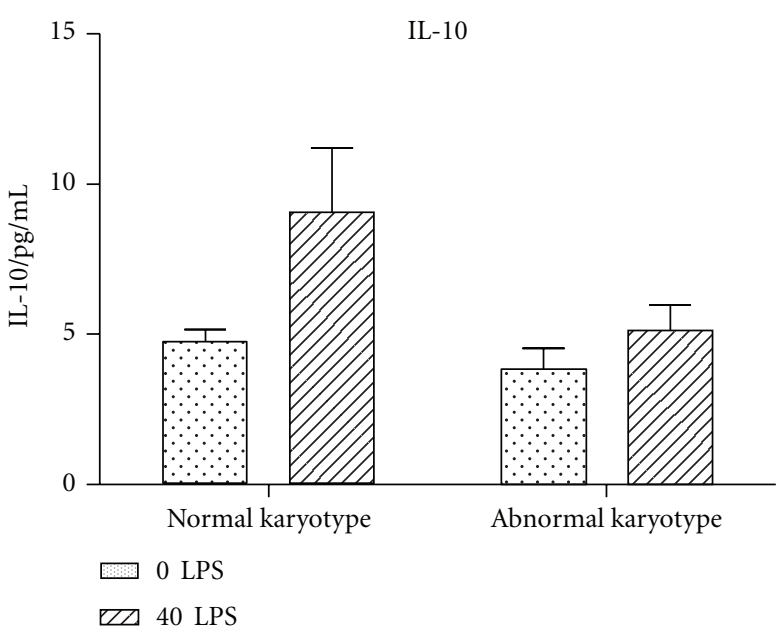

(b)

Figure 2: Levels of cytokines in monocytes ( $n=12$ with normal karyotype and $n=14$ with abnormal karyotype). There was no significant difference in any of the intracellular levels of cytokines.

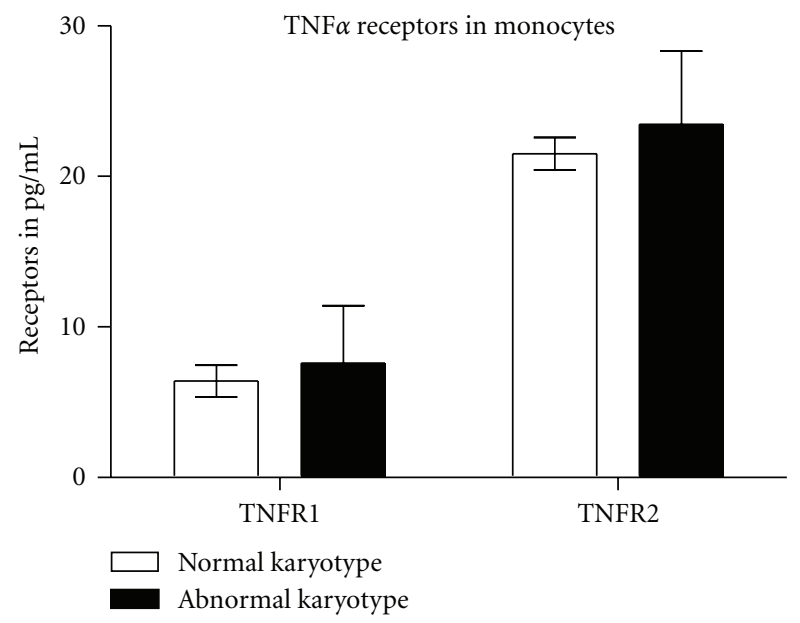

FIGURE 3: Levels of TNF-R1 and TNF-R2 in monocytes $(n=12$ with normal karyotype and $n=14$ with abnormal karyotype). There was no significant difference in any of the intracellular levels of receptors.

maternal systemic inflammatory response with an imbalance in the Th-1/Th-2 ratio in the maternal circulation. By contrast, in villous samples from the same groups, we found an increased secretion of inflammatory cytokine TNF $\alpha$ and its soluble receptors, TNF-R1 and TNF-R2, and the antiinflammatory cytokine IL-10 in cases with an abnormal karyotype, compared to those presenting with a normal karyotype.

We have previously shown that in early pregnancy failure, the excessive entry of oxygenated maternal blood into the intervillous space has a direct mechanical effect on the villous tissue which becomes enmeshed inside large intervillous blood thrombi and an indirect $\mathrm{O}_{2}$-mediated trophoblastic damage with increased apoptosis [21-23]. This phenomenon occurs at some point in miscarriages independently of the aetiology and in particular of the conceptus karyotype [24]. As all our villous tissue samples were obtained from missed miscarriage, the abnormal influx of maternal oxygenated blood would have had a similar impact on the villous tissue of both the cases presenting with and those presenting without a chromosomal karyotype at the time of the clinical diagnosis. This can explain why $\mathrm{O}_{2}$ and the number of days in culture had no impact on cytokine and receptor levels in our explant cultures experiments in both groups. The absence of significant difference in cytokine levels in the snap frozen samples (on day of collection) suggests that the difference seen in culture reflects functional changes.

In the present study, we also found that in early pregnancy (i.e., before 14 weeks), the amount of TNF $\alpha$ and IL-10 secreted in vitro by villous tissue from sporadic miscarriage was lower in the group with a normal karyotype. These data indicate that the karyotype of the conceptus has a direct impact on the secretion of cytokines by the villous tissue. An abnormal karyotype leads to an elevated local inflammatory response, confirmed by the significant rise in TNF $\alpha$. This was accompanied by a rise in the antiinflammatory cytokine IL-10 and the neutralizing soluble TNF-R1 and TNF-R2. An upregulation of the cytokine expression by the villous tissue has been reported in many cases of unexplained spontaneous miscarriages associated with a severe congenital infection presenting with maternal systemic symptoms [25]. In early miscarriages, there is massive destruction of maternal immunoglobulins in embryonic monocytes, with acute villitis in the placental barrier [26]. Some authors have therefore suggested that the upregulation of cytokines is deleterious to the developing placenta and fetus, probably as a consequence of vasoconstriction and/or direct cellular damage $[27,28]$. Chromosomally abnormal spontaneous miscarriages may occur because of different mechanisms than chromosomally normal spontaneous miscarriages. Chromosome aberrations cause changes in placental morphology and function, including size, shape, 
and vascularity and may affect rates of apoptosis of the stromal cells and cell proliferation in blood vessels during differentiation of chorionic villi [29].

In the maternal plasma, we observed inflammatory changes in the opposite direction than in villous tissue. The group presenting with a normal karyotype had a shift towards a Th-1 cytokine immune response in the circulation, reflected by a higher TNF $\alpha /$ IL-10 cytokine ratio than miscarriages with abnormal karyotype. Monocytes, in the normal karyotype group released a higher level of TNF $\alpha$ upon stimulation with LPS. This suggests that in the case of miscarriage of a karyotypically normal fetus, the maternal systemic inflammatory response is more sensitized. It is possible that in the case of karyotypically normal miscarriage, there is a systemic maternal immune response that causes the rejection of the fetus. In contrast, in cases with an abnormal karyotype, implantation would have already failed following the local excessive inflammatory reaction at the materno-fetal placental interface.

In recurrent miscarriage, the circulating cytokine levels and the decidual cytokine profile are different from those found in normal first trimester controls [30-33]. In particular, during periconception in recurrent miscarriage, higher circulatory levels of NK cells [34] and higher serum levels of macrophage migration inhibition factor [35] have been shown to be predictive of miscarriage of a conceptus with normal karyotype. In addition, women with recurrent miscarriages who miscarry a pregnancy with normal karyotype have decreased serum levels of TNF $\alpha$ [36] as early as 6-7 weeks of gestation. In our study, we have only looked at spontaneous sporadic miscarriages; however, miscarriages of normal karyotype are more likely to recur due to a possible underlying maternal immune problem [37].

The biological functions TNF $\alpha$ depends on its binding to two known receptors, TNF-R1 and TNF-R2 [38]. The soluble forms of these membrane receptors bind to TNF $\alpha$ with high affinity and can neutralize $\mathrm{TNF} \alpha$ function [39, 40]. Yu et al., using flowcytometric and immunohistochemical measurement of TNF-R1 in the decidua, and detection of serum levels of soluble TNF-R1 by enzymelinked immunoassays, found an association between the over-expression of TNF-R1 and early pregnancy failure [41]. In our study, villous secretion of soluble TNF-R1 in the culture medium and soluble TNF-R2 content in villous homogenate was significantly higher in cases presenting with abnormal karyotype.

Our data illustrate that the mechanisms leading to a miscarriage may depend on the karyotype of the conceptus. We suggest that there is a local functional disturbance in the karyotypically abnormal placental tissue while, in the case of a normal karyotype miscarriage, rejection occurs due to a maternal systemic inflammation.

\section{Authors' Contribution}

E. Jauniaux and S. Muttukrishna have equal contribution. J. Calleja-Agius collected the samples and performed the experiments and statistical analysis and wrote the first draft of the manuscript. S. Muttukrishna and E. Jauniaux contributed to the study design interpretation of the data and writing of the manuscript. S. Muttukrishna supervised the laboratory work and data analysis.

\section{Ethical Approval}

The procedures of this study received ethical approval from the the UCL Ethics Committee at The National Hospital for Neurology and Neurosurgery and Institute of Neurology Joint Research Ethics Committee. This research work is part of project entitled "Early Pregnancy Complications: What causes them and how can we improve diagnosis?" (Project ID: 07/QO512/41) and was ethically approved in April 2008.

\section{References}

[1] E. R. Norwitz, D. J. Schust, and S. J. Fisher, "Implantation and the survival of early pregnancy," New England Journal of Medicine, vol. 345, no. 19, pp. 1400-1408, 2001.

[2] J. L. Simpson, "Incidence and timing of pregnancy losses: relevance to evaluating safety of early prenatal diagnosis," American Journal of Medical Genetics, vol. 35, no. 2, pp. 165$173,1990$.

[3] J. L. Simpson, J. L. Mills, H. Kim et al., "Infectious processes: an infrequent cause of first trimester spontaneous abortions," Human Reproduction, vol. 11, no. 3, pp. 668-672, 1996.

[4] E. Ljunger, S. Cnattingius, C. Lundin, and G. Annerén, "Chromosomal anomalies in first-trimester miscarriages," Acta Obstetricia et Gynecologica Scandinavica, vol. 84, no. 11, pp. 1103-1107, 2005.

[5] E. Jauniaux and G. J. Burton, "Pathophysiology of histological changes in early pregnancy loss," Placenta, vol. 26, no. 2-3, pp. 114-123, 2005.

[6] J. Hustin, E. Jauniaux, and J. P. Schaaps, "Histological study of the materno-embryonic interface in spontaneous abortion," Placenta, vol. 11, no. 6, pp. 477-486, 1990.

[7] E. Jauniaux, J. Zaidi, D. Jurkovic, S. Campbell, and J. Hustin, "Comparison of colour Doppler features and pathological findings in complicated early pregnancy," Human Reproduction, vol. 9, no. 12, pp. 2432-2437, 1994.

[8] R. Shurtz-Swirski, R. J. Simon, Y. Cohen, and E. R. Barnea, "Human embryo modulates placental function in the first trimester; effects of neural tissues upon chorionic gonadotropin and progesterone secretion," Placenta, vol. 12, no. 5, pp. 521-532, 1991.

[9] J. R. Challis, C. J. Lockwood, L. Myatt, J. E. Norman, J. F. Strauss III, and F. Petraglia, "Inflammation and pregnancy," Reproductive Sciences, vol. 16, no. 2, pp. 206-215, 2009.

[10] O. B. Christiansen, H. S. Nielsen, and A. M. Kolte, "Inflammation and miscarriage," Seminars in Fetal and Neonatal Medicine, vol. 11, no. 5, pp. 302-308, 2006.

[11] C. A. Koch and J. L. Platt, "T cell recognition and immunity in the fetus and mother," Cellular Immunology, vol. 248, no. 1, pp. 12-17, 2007.

[12] S. Bauer, J. Pollheimer, J. Hartmann, P. Husslein, J. D. Aplin, and M. Knöfler, "Tumor necrosis factor-alpha inhibits trophoblast migration through elevation of plasminogen activator inhibitor-1 in first-trimester villous explant cultures," Journal of Clinical Endocrinology and Metabolism, vol. 89, no. 2, pp. 812-822, 2004.

[13] F. Monzón-Bordonaba, F. Vadillo-Ortega, and R. F. Feinberg, "Modulation of trophoblast function by tumor necrosis factor- $\alpha$ : a role in pregnancy establishment and maintenance?" 
American Journal of Obstetrics and Gynecology, vol. 187, no. 6, pp. 1574-1580, 2002.

[14] O. Blanco, I. Tirado, R. Muñoz-Fernández et al., "Human decidual stromal cells express HLA-G Effects of cytokines and decidualization," Human Reproduction, vol. 23, no. 1, pp. 144$152,2008$.

[15] K. J. H. Lim, O. A. Odukoya, R. A. Ajjan, T. C. Li, A. P. Weetman, and I. D. Cooke, "The role of T-helper cytokines in human reproduction," Fertility and Sterility, vol. 73, no. 1, pp. 136-142, 2000.

[16] O. V. Pavlov, D. V. Lalayan, and S. A. Sel'kov, "Spontaneous and LPS-induced secretion of cytokines by villous chorion tissue," Bulletin of Experimental Biology and Medicine, vol. 141, no. 6, pp. 720-723, 2006.

[17] S. Banerjee, A. Smallwood, J. Moorhead et al., "Placental expression of interferon- $\gamma$ (IFN- $\gamma$ ) and its receptor IFN- $\gamma$ R2 fail to switch from early hypoxic to late normotensive development in preeclampsia," Journal of Clinical Endocrinology and Metabolism, vol. 90, no. 2, pp. 944-952, 2005.

[18] J. Calleja-Agius, S. Muttukrishna, A. R. Pizzey, and E. Jauniaux, "Pro- and antiinflammatory cytokines in threatened miscarriages," American Journal of Obstetrics and Gynecology, vol. 205, no. 1, pp. 83.e8-83.e16, 2011.

[19] D. E. Rooney, Human Cytogenetics: Constitutional Analysis, Oxford University Press, Oxford, UK, 3rd edition, 2001.

[20] A. Khalil, E. Jauniaux, K. Harrington, and S. Muttukrishna, "Placental production and maternal serum and urine levels of inhibin A and activin A are modified by antihypertensive therapy in hypertensive disorders of pregnancy," Clinical Endocrinology, vol. 70, no. 6, pp. 924-931, 2009.

[21] E. Jauniaux, J. Hempstock, N. Greenwold, and G. J. Burton, "Trophoblastic oxidative stress in relation to temporal and regional differences in maternal placental blood flow in normal and abnormal early pregnancies," American Journal of Pathology, vol. 162, no. 1, pp. 115-125, 2003.

[22] K. Kokawa, T. Shikone, and R. Nakano, "Apoptosis in human chorionic villi and decidua during normal embryonic development and spontaneous abortion in the first trimester," Placenta, vol. 19, no. 1, pp. 21-26, 1998.

[23] J. Hempstock, E. Jauniaux, N. Greenwold, and G. J. Burton, "The contribution of placental oxidative stress to early pregnancy failure," Human Pathology, vol. 34, no. 12, pp. 12651275, 2003.

[24] E. Jauniaux, L. Poston, and G. J. Burton, "Placental-related diseases of pregnancy: involvement of oxidative stress and implications in human evolution," Human Reproduction Update, vol. 12, no. 6, pp. 747-755, 2006.

[25] G. J. Nuovo, L. D. Cooper, and D. Bartholomew, "Histologic, infectious, and molecular correlates of idiopathic spontaneous abortion and perinatal mortality," Diagnostic Molecular Pathology, vol. 14, no. 3, pp. 152-158, 2005.

[26] P. Gurevich, A. Elhayany, A. P. Milovanov et al., "The placental barrier in allogenic immune conflict in spontaneous early abortions: immunohistochemical and morphological study," American Journal of Reproductive Immunology, vol. 58, no. 5, pp. 460-467, 2007.

[27] H. Kadhim, B. Tabarki, C. De Prez, and G. Sébire, "Cytokine immunoreactivity in cortical and subcortical neurons in periventricular leukomalacia: are cytokines implicated in neuronal dysfunction in cerebral palsy?" Acta Neuropathologica, vol. 105, no. 3, pp. 209-216, 2003.

[28] L. F. Shalak, A. R. Laptook, H. S. Jafri, O. Ramilo, and J. M. Perlman, "Clinical chorioamnionitis, elevated cytokines, and brain injury in term infants," Pediatrics, vol. 110, no. 4, pp. 673-680, 2002.
[29] M. B. Qumsiyeh, K. R. Kim, M. N. Ahmed, and W. Bradford, "Cytogenetics and mechanisms of spontaneous abortions: increased apoptosis and decreased cell proliferation in chromosomally abnormal villi," Cytogenetics and Cell Genetics, vol. 88, no. 3-4, pp. 230-235, 2000.

[30] C. Jenkins, R. Wilson, J. Roberts, H. Miller, J. H. McKillop, and J. J. Walker, "Antioxidants: their role in pregnancy and miscarriage," Antioxidants and Redox Signaling, vol. 2, no. 3, pp. 623-628, 2000.

[31] M. Makhseed, R. Raghupathy, F. Azizieh, R. Farhat, N. Hassan, and A. Bandar, "Circulating cytokines and CD30 in normal human pregnancy and recurrent spontaneous abortions," Human Reproduction, vol. 15, no. 9, pp. 2011-2017, 2000.

[32] M. Von Wolff, C. J. Thaler, T. Strowitzki, J. Broome, W. Stolz, and S. Tabibzadeh, "Regulated expression of cytokines in human endometrium throughout the menstrual cycle: dysregulation in habitual abortion," Molecular Human Reproduction, vol. 6, no. 7, pp. 627-634, 2000.

[33] N. Baxter, M. Sumiya, S. Cheng et al., "Recurrent miscarriage and variant alleles of mannose binding lectin, tumour necrosis factor and lymphotoxin $\alpha$ genes," Clinical and Experimental Immunology, vol. 126, no. 3, pp. 529-534, 2001.

[34] H. Yamada, M. Morikawa, E. H. Kato, S. Shimada, G. Kobashi, and H. Minakami, "Pre-conceptional natural killer cell activity and percentage as predictors of biochemical pregnancy and spontaneous abortion with normal chromosome karyotype," American Journal of Reproductive Immunology, vol. 50, no. 4, pp. 351-354, 2003.

[35] H. Yamada, E. H. Kato, M. Morikawa et al., "Decreased serum levels of macrophage migration inhibition factor in miscarriages with normal chromosome karyotype," Human Reproduction, vol. 18, no. 3, pp. 616-620, 2003.

[36] H. Yamada, M. Morikawa, I. Furuta et al., "Circulating cytokines during early pregnancy in women with recurrent spontaneous abortion: decreased TNF-alpha levels in abortion with normal chromosome karyotype," The Hokkaido Journal of Medical Science, vol. 79, no. 3, pp. 237-241, 2004.

[37] H. Carp, V. Toder, A. Aviram, M. Daniely, S. Mashiach, and G. Barkai, "Karyotype of the abortus in recurrent miscarriage," Fertility and Sterility, vol. 75, no. 4, pp. 678-682, 2001.

[38] H. Takada, N. J. Chen, C. Mirtsos et al., "Role of SODD in regulation of tumor necrosis factor responses," Molecular and Cellular Biology, vol. 23, no. 11, pp. 4026-4033, 2003.

[39] P. Reddy, J. L. Slack, R. Davis et al., "Functional analysis of the domain structure of tumor necrosis factor- $\alpha$ converting enzyme," Journal of Biological Chemistry, vol. 275, no. 19, pp. 14608-14614, 2000.

[40] C. A. Buckley, F. N. Rouhani, M. Kaler, B. Adamik, F. I. Hawari, and S. J. Levine, "Amino-terminal TACE prodomain attenuates TNFR2 cleavage independently of the cysteine switch," American Journal of Physiology, vol. 288, no. 6, pp. L1132-L1138, 2005.

[41] X. W. Yu, C. F. Yan, H. Jin, and X. Li, "Tumor necrosis factor receptor 1 expression and early spontaneous abortion," International Journal of Gynecology and Obstetrics, vol. 88, no. 1, pp. 44-48, 2005. 




The Scientific World Journal
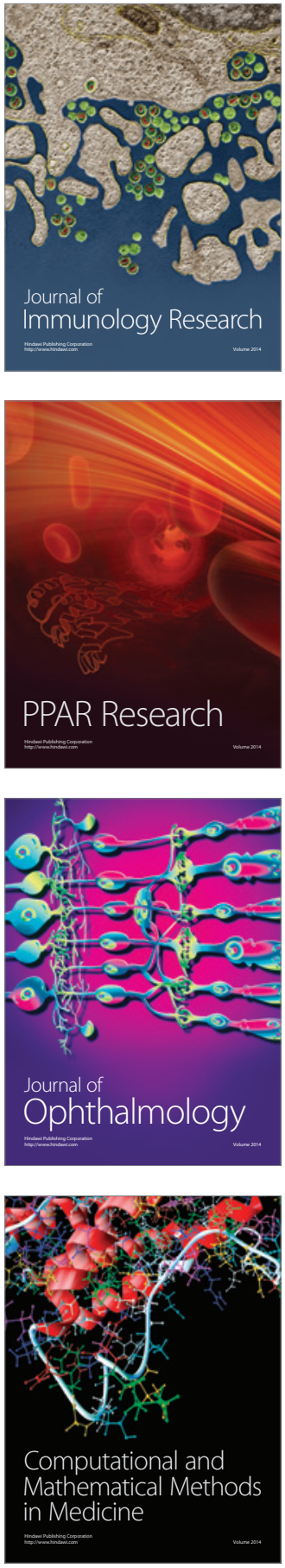

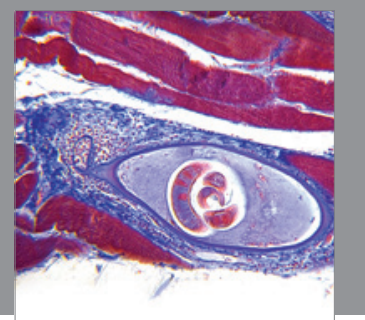

Gastroenterology

Research and Practice
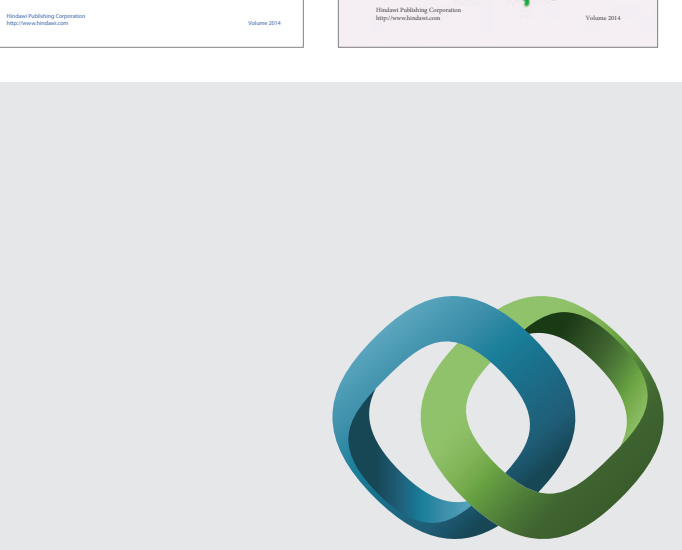

\section{Hindawi}

Submit your manuscripts at

http://www.hindawi.com
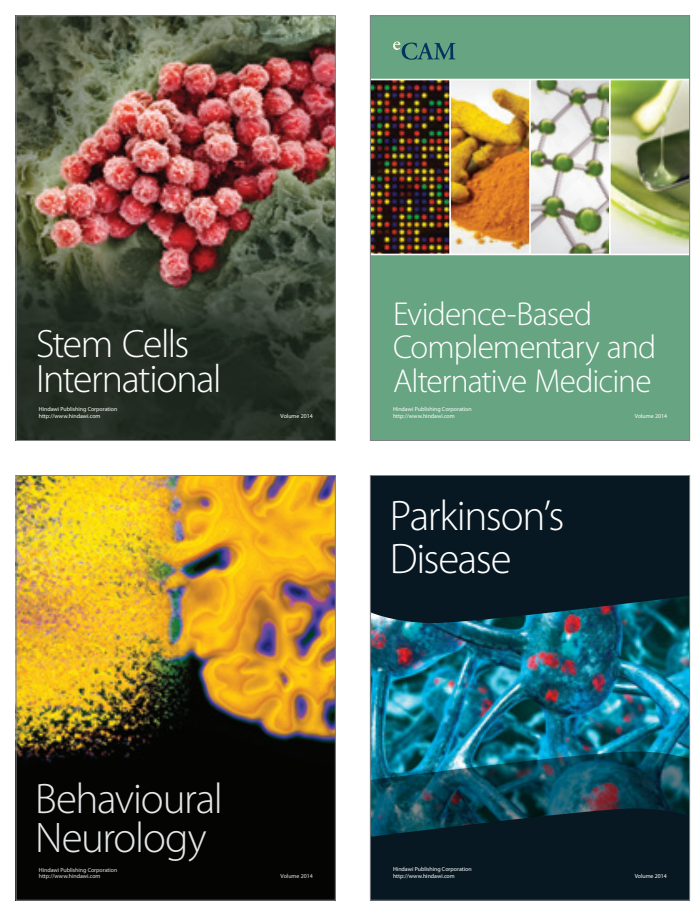

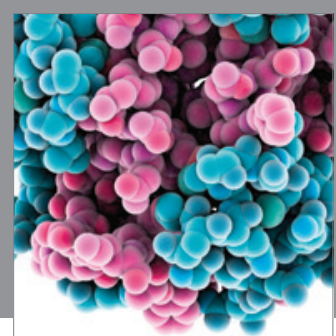

Journal of
Diabetes Research

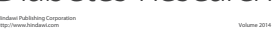

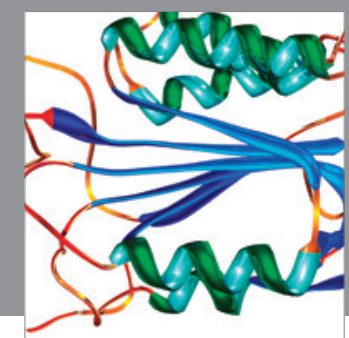

Disease Markers
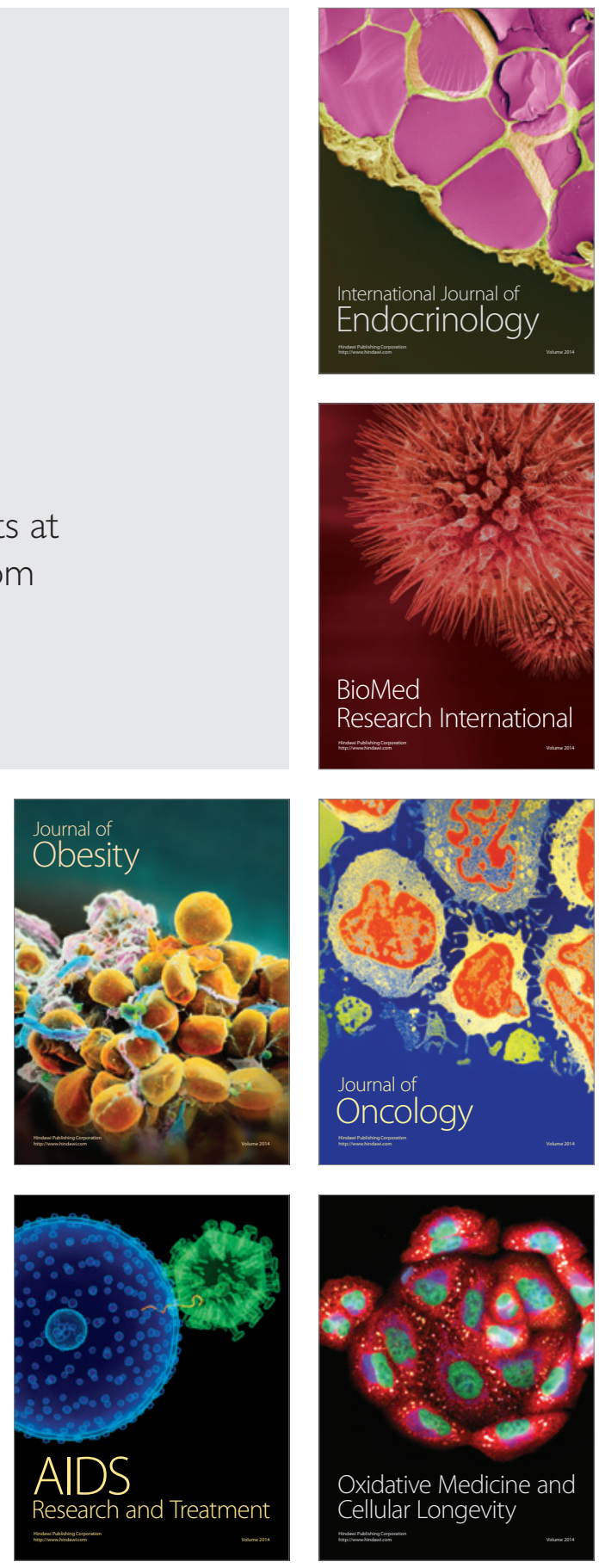